\title{
Hybrid Fibers Transform into Distinct Fiber Types in Maturing Mouse Muscles
}

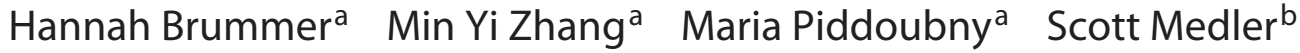

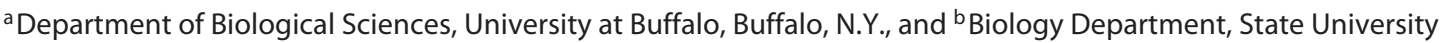 \\ of New York at Fredonia, Fredonia, N.Y., USA
}

\section{Key Words}

Skeletal muscle $\cdot$ Fiber type $\cdot$ Hybrid fibers $\cdot$ Myosin heavy chain $\cdot$ Muscle development

\begin{abstract}
The role of hybrid fibers as intermediates in fiber type transformations is not completely understood. In some cases hybrids are clearly transitional fibers changing from one type to another, but in others they represent phenotypically stable fibers in normal muscles. In the current study, our goal was to understand the fate of hybrid fibers in fiber type transitions that take place during muscle maturation. Previous studies have reported high proportions of hybrid fibers during postnatal development, but few have followed the fate of these fibers past the time of weaning. We quantified proportions of hybrid fibers in three different mouse skeletal muscles from newly weaned to 6-month-old mice. Hybrid fibers were very prevalent in the brachioradialis (BR) and tibialis anterior (TA) muscles from newly weaned mice, where they constituted 50 and $40 \%$ of the fibers, respectively. These hybrids declined steadily to about $15-30 \%$ over the next several months. In the soleus muscle the proportion of hybrids did not change, but constituted approximately $20 \%$ of fibers. The reduction in IIX/IIB hybrids resulted from different processes in the $B R$ and the TA. In the $B R$, the reduction was coincident with an increase in type IIX fibers. In the TA, the number of IIX/IIB hybrids was inversely correlated with
\end{abstract}

the proportion of IIB fibers. These patterns reveal that the role of hybrid fibers as intermediates in muscle development is complex. Some hybrid fibers in maturing muscles represent transitional fiber types, while others are phenotypically stable. Moreover, the fate of transitional fibers may be distinct among similar fiber types within different muscles.

(c) 2013 S. Karger AG, Basel

\section{Introduction}

Skeletal muscles are comprised of hundreds to thousands of individual muscle fibers that exist in several types, as identified by the expression of alternate isoforms of myosin heavy chain (MHC) and other myofibrillar proteins [Schiaffino and Reggiani, 2011]. In the limb muscles of small mammals, four MHC isoforms are expressed and provide a continuum of shortening speeds from slowest to fastest in the order: I $\rightarrow$ IIA $\rightarrow$ IIX $\rightarrow$ IIB [Schiaffino and

\begin{tabular}{ll} 
Abbreviations used in this paper \\
\hline BR & $\begin{array}{l}\text { brachioradialis } \\
\text { myosin heavy chain }\end{array}$ \\
MHC & $\begin{array}{l}\text { sodium dodecyl sulfate-polyacrylamide } \\
\text { gel electrophoresis }\end{array}$ \\
SOL & soleus \\
TA & tibialis anterior
\end{tabular}

Dr. Scott Medler

Biology Department, State University of New York at Fredonia 205 Jewett Hall

Fredonia, NY 14063 (USA)

E-Mail Scott.Medler@fredonia.edu 
Reggiani, 1996, 2011]. Over the past several decades, we have gained an ever-increasing understanding of the diversity and complexity of individual fiber types. Fiber types that were once defined simply as 'fast' or 'slow' are in reality part of a continuum of fiber types that are difficult to classify into precise categories [Staron and Pette, 1993; Caiozzo et al., 2003; Medler and Mykles, 2003; Medler et al., 2004; Zhang et al., 2010; Schiaffino and Reggiani, 2011]. During development, fibers undergo major transitions in MHC isoform expression, with developmental isoforms being exchanged for adult MHCs [Di Maso et al., 2000; Agbulut et al., 2003; Lieber, 2010]. Even fully differentiated adult skeletal muscles maintain the capacity to adapt to a variety of external stimuli through phenotypic plasticity [Pette and Staron, 2001; Schiaffino and Reggiani, 2011]. In the most dramatic cases, individual fibers may completely transform from one fiber type to another. This complex process requires a major shift in the expression of MHC and other myofibrillar proteins, as well as the selective proteolysis and removal of the existing isoforms. During this process of fiber type switching, fibers temporarily become hybrids, co-expressing two or more MHC isoforms within the same fiber.

Fiber type transitions are especially important during periods of muscle differentiation and development. Early on, embryonic and neonatal MHCs are expressed to the exclusion of the adult isoforms, and these isoforms continue to be expressed along with adult MHCs for the first weeks after birth [Di Maso et al., 2000; Agbulut et al., 2003]. Adult MHC isoforms are present in skeletal muscles by the time of birth, but transitions in fiber type continue as the muscles grow and mature [Wigston and English, 1992; Agbulut et al., 2003]. During this postnatal period, hybrid fibers containing mixtures of developmental and adult MHC isoforms are common [Wigston and English, 1992; Di Maso et al., 2000]. In mouse muscles, developmental MHC isoform expression ends by postnatal day 21, but some shifts in the proportions of the adult $\mathrm{MHC}$ isoforms continue for several months [Agbulut et al., 2003]. In the rat soleus (SOL) muscle, hybrids expressing both fast and slow MHCs decrease significantly over the first year of development, as most of these fibers are transformed into pure slow fibers [Wigston and English, 1992]. In mouse SOL, some level of transformation takes place over the same time period, but is much less dramatic [Wigston and English, 1992].

While hybrid fibers can provide an indication of such fiber type transitions [Talmadge et al., 1995, 1999; Di Maso et al., 2000; Unguez et al., 2000; Williamson et al., 2000], in many instances hybrid fibers persist as stable fiber pheno- types [Stephenson, 2001; Caiozzo et al., 2003; Acevedo and Rivero, 2006; Glaser et al., 2010]. In human muscles, the proportion of hybrid fibers is variable, but on average about 15-20\% hybrid fibers is normal [Andersen et al., 1999; Williamson et al., 2000; Andersen, 2003]. In rat muscles hybrid fibers are especially common, with many muscles being comprised of 50-75\% hybrid fibers [Caiozzo et al., 2003]. In mouse, the overall fiber type distribution is lower than in the rat, being highly skewed toward the fastest IIB fiber type. Nevertheless, certain mouse muscles contain $25-50 \%$ of their fibers as hybrids [Glaser et al., 2010; Zhang et al., 2010]. In the muscles of both of these rodents, the most prevalent hybrid fiber types are the IIX/IIB hybrids, which represent roughly $70-90 \%$ of all the hybrid fibers [Caiozzo et al., 2003; Glaser et al., 2010; Zhang et al., 2010].

In the current study, we used single fiber analysis to precisely identify hybrid fiber types in three muscles following weaning. We determined $\mathrm{MHC}$ isoform content in single fibers from the brachioradialis (BR), tibialis anterior (TA) and SOL muscles from newly weaned mice through animals of approximately 6 months of age. Our goal was to determine what role hybrid fibers play in the maturation of selected mouse muscles in the weeks following weaning. Our hypothesis was that the proportion of hybrid fibers would decline as they were converted into pure fiber types during the process of the skeletal muscle maturation. The BR and TA were selected because we have previously identified these muscles as expressing high proportions of hybrid fibers [Zhang et al., 2010]. The SOL was selected because it contains a population of hybrids co-expressing type I and IIA MHC isoforms, and has been the focus of previous studies of mouse muscle development [Wigston and English, 1992; Agbulut et al., 2003]. Briefly, we found that the proportion of hybrid fibers decreased significantly over the first several weeks following weaning in the BR and TA muscles, but that the proportion of hybrids was stable in the SOL during this same time period. The IIX/IIB hybrids that were lost during muscle maturation had different fates in the BR and the TA muscles. In the BR most of the transitional hybrids were converted into IIX fibers, while in the TA the hybrid fibers predominantly transitioned into IIB fibers.

\section{Materials and Methods}

Animals

Mice (C57BL/6J) were purchased from Jackson Laboratory (Bar Harbor, Me., USA) and were maintained in accordance with an IACUC-approved protocol at the University at Buffalo. They were housed in cages with a 12-hour-12-hour light-dark cycle and 
were provided with food and water ad libitum. Litters were kept with their mothers until 21 days of age when they were separated with their same sex littermates into their own cages. Mice were sacrificed at different ages ranging from 23 to 210 days old. Fibers were collected from 12 male or female mice.

\section{Single Fiber Analyses}

Mice were euthanized by exposure to $\mathrm{CO}_{2}$ and muscles were collected immediately. Whole muscles were transferred to an ice-cold relaxing solution ( $50 \%$ glycerol, $2 \mathrm{mM}$ EGTA, $1 \mathrm{mM} \mathrm{MgCl}_{2}, 4 \mathrm{mM}$ ATP, $10 \mathrm{mM}$ imidazole and $100 \mathrm{mM} \mathrm{KCl}$ ) and stored at $-20^{\circ} \mathrm{C}$. Individual fibers were isolated in cold relaxing solution with fine forceps under a stereomicroscope. A total of 2,587 fibers were analyzed in this study (818 SOL, 723 BR and 1,046 TA), with approximately 100 fibers sampled per animal. Each fiber was placed into a microcentrifuge tube containing $\sim 30 \mu$ of sample buffer containing $8 \mathrm{M}$ urea, $2 \mathrm{M}$ thiourea, $50 \mathrm{mM}$ Tris base, $75 \mathrm{mM}$ dithiothreitol, $3 \%$ sodium dodecyl sulfate (SDS) and $0.004 \%$ bromophenol blue, pH 6.8 [Blough et al., 1996; Zhang et al., 2010]. Samples were heated to $65^{\circ} \mathrm{C}$ for $15 \mathrm{~min}$ and $10 \mu \mathrm{l}$ of each sample was applied to the gel. SDS polyacrylamide gel electrophoresis (SDS-PAGE) was used with a Hoeffer SE 600 to resolve individual isoforms of MHC. Resolving gels consisted of 12\% acrylamide (200:1 acrylamide:methylenebis-acrylamide), $12 \%$ glycerol, $0.675 \mathrm{M}$ Tris base ( $\mathrm{pH} 8.8$ ) and $0.1 \%$ SDS. Stacking gels consisted of $4 \%$ acrylamide (20:1 acrylamide: methylene-bis-acrylamide), $0.125 \mathrm{M}$ Tris base ( $\mathrm{pH}$ 6.8) and $0.1 \%$ SDS. The running buffer contained $0.192 \mathrm{M}$ glycine, $25 \mathrm{mM}$ Tris base, $0.1 \%$ SDS and $0.08 \% 2$-mercaptoethanol. Gels were run with a constant current of $20 \mathrm{~mA}$ for approximately $41 \mathrm{~h}$ at $8^{\circ} \mathrm{C}$. At the end of the run, gels were fixed in $50 \%$ methanol that included formaldehyde $(0.037 \%)$ to increase the sensitivity of the silver staining procedure. Gels were allowed to fix overnight in this solution and then washed for $1 \mathrm{~h}$ in deionized water before staining with silver. Gels were then stained with an ammoniacal silver staining procedure [Wray et al., 1981; Medler et al., 2007].

Dried gels were scanned at high resolution and the relative proportions of $\mathrm{MHC}$ isoforms in single fibers were determined from digital images using densitometry (Image J 1.42q, NIH). Serial dilutions of standards containing known proportions of $\mathrm{MHC}$ isoforms were used to establish that concentrations were a linear function of sample density.

\section{Segmental Expression of MHC Isoforms}

Individual fibers from the muscles studied averaged about 2$3 \mathrm{~mm}$ in length after they were dissected from the whole muscle. We cut these individual fibers into 3 approximately equal lengths and placed each segment in an individual tube with urea sample buffer. For these analyses we focused on TA and BR fibers from newly weaned animals because we had already identified these muscles as containing high proportions of IIX/IIB hybrids. Samples were separated using SDS-PAGE as described above, and the specific MHC isoforms within each segment were identified. In identified hybrids, we used densitometry to quantify the relative proportions of the IIX and IIB isoforms to identify differences in $\mathrm{MHC}$ expression along the lengths of single fibers.

\section{Statistical Analyses}

Fiber proportions within muscles were analyzed as a function of animal age (days from birth) using regression analyses. Power regression models were fit to the data because the drops in hybrid

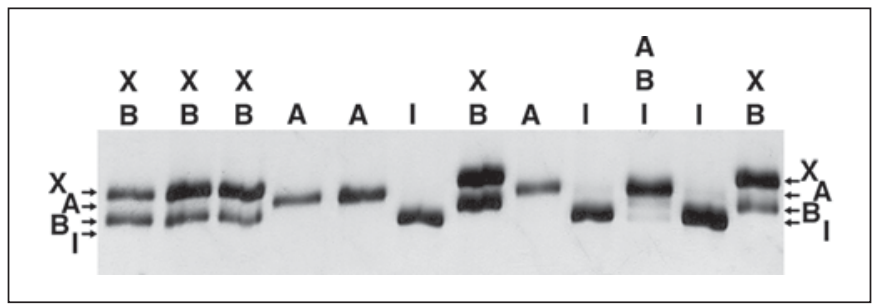

Fig. 1. Mouse fiber types identified through SDS-PAGE of proteins from individual fibers. Using the described SDS-PAGE conditions, the $\mathrm{MHC}$ isoforms migrate as I > IIB > IIA > IIX. Single fibers shown here include a number of different fiber types, including several IIX/IIB hybrids. Deduced fiber types are indicated above each lane in the gel.

fibers within the TA and BR were clearly curvilinear, and these models fit the data better than simple linear regression. We also used linear regression to test for other correlations among fiber type proportions (IIX, IIB and IIX/IIB), and for correlations between fiber types and animal age or mass. Relative proportions of IIX and IIB isoforms within IIX/IIB hybrids were also analyzed as a function of developmental age.

In addition to using regression to track hybrid proportions as a continuous function of age, we also assessed differences in hybrid fibers between newly weaned versus mature mouse muscles. Average proportions of specific MHC isoforms in these muscles were compared using t tests. Critical $p$ values for significant differences were adjusted with a Bonferroni adjustment to set the experimentwise error rate to 0.05 .

\section{Results}

Proportions of Fiber Types in TA, BR and SOL Muscles Under our gel parameters and running conditions, the four MHC isoforms migrated in the order I > IIB > IIA > IIX (fig. 1). This pattern is different from the results reported for rodent MHCs in other studies [Talmadge and Roy, 1993; Di Maso et al., 2000; Caiozzo et al., 2003; Glaser et al., 2010] in that the IIA and IIX isoforms are reversed in their migration order. As we have indicated previously, the result is better separation of the IIA and IIX isoforms [Zhang et al., 2010]. In both the TA and BR muscles, the vast majority of fibers were IIX, IIB or IIX/IIB hybrids (the sum of these fiber types totaled $97.4 \pm 0.93 \%$ and $95.9 \pm 1.38 \%$ in the TA and BR, respectively; fig. 2). About $2 \%$ of the fibers were IIA/IIX hybrids in both muscles, followed by minor amounts of other fiber types (fig. 2). In the SOL, the type I and IIA fibers were dominant, followed by I/IIA hybrids. Approximately $4 \%$ of the fibers were IIX, 4\% were IIX/IIB hybrids, followed by about 2\% IIA/IIX hybrids (fig. 2). 


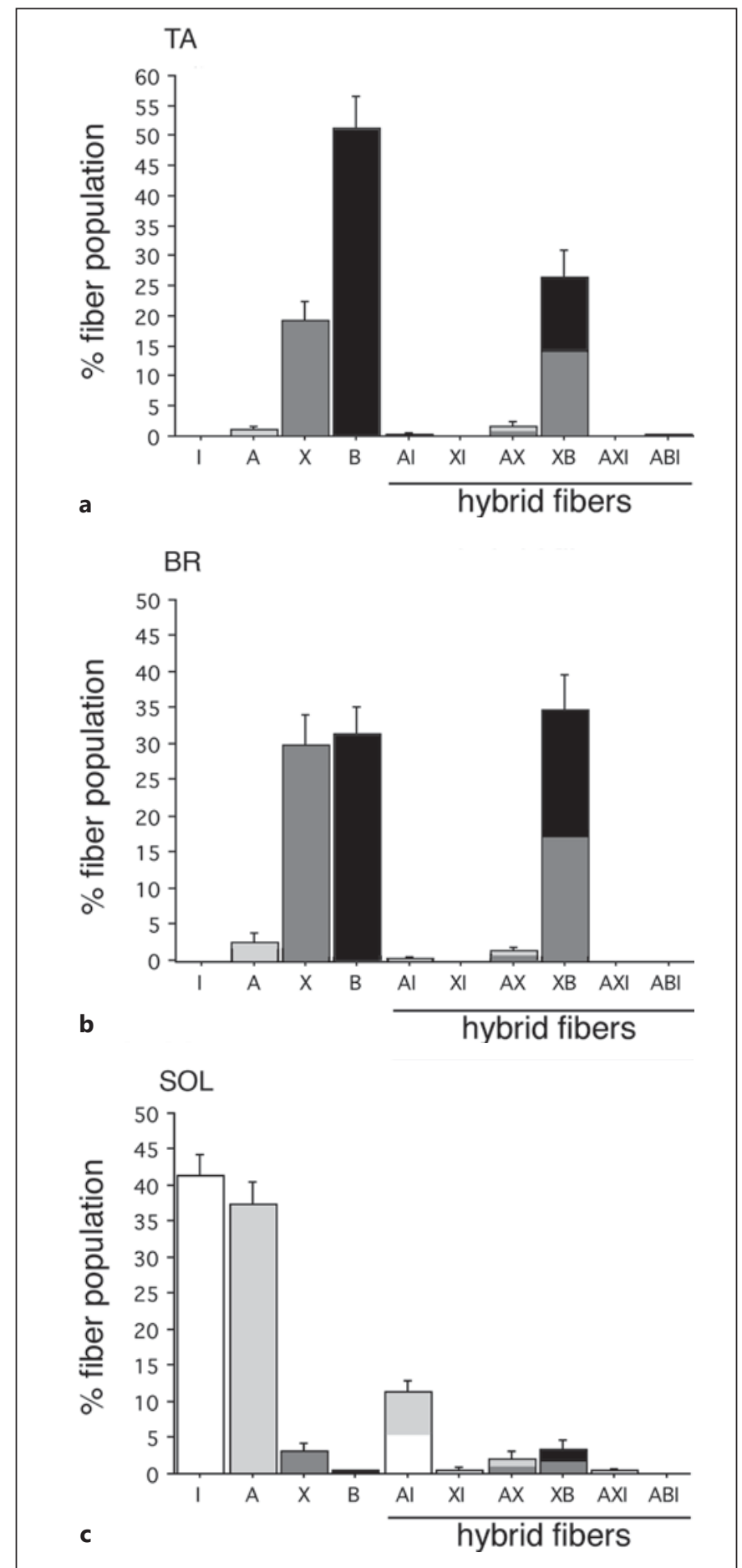

Fig. 2. Overall fiber type distributions in the TA, BR and SOL muscles averaged for all mice. a In the TA the predominant isoform was IIB, followed by IIX and IIX/IIB. $\mathbf{b}$ In the BR the IIB, IIX and IIX/IIB fiber types were present in nearly equal proportions. c In the SOL the type I and IIA isoforms were present in similar proportions and the I/IIA fibers were the predominant hybrid fiber type. In each muscle, minor amounts of other fiber types were present.

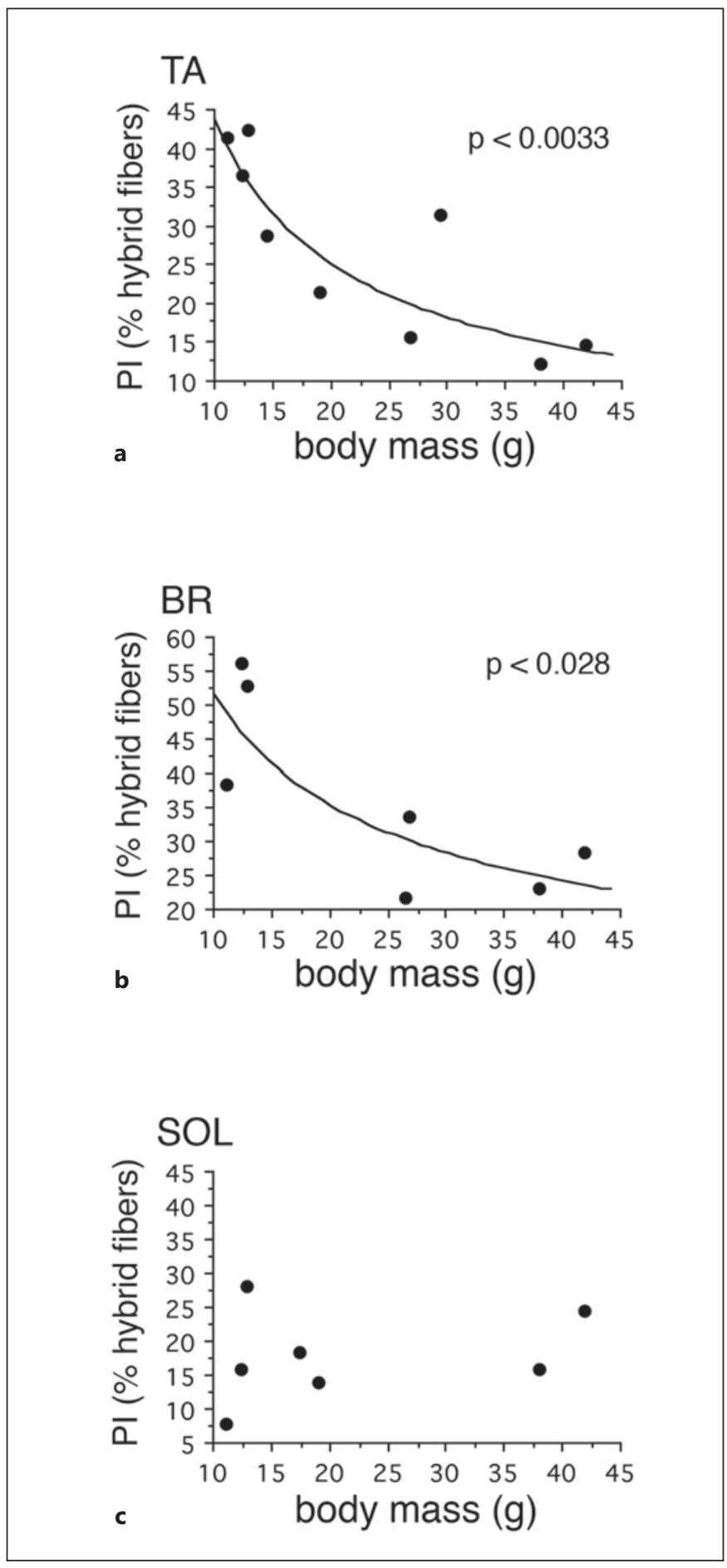

Fig. 3. Changes in hybrid fiber proportions as a function of mouse body mass. a The TA muscle exhibited a significant curvilinear decline in hybrid fibers with increased mass $(\mathrm{p}<0.0033)$. b The BR muscle similarly showed a curvilinear reduction in hybrid fiber proportions with growth $(\mathrm{p}<0.028)$. c The proportions of hybrid fibers within the SOL muscle were stable over time at 15-20\%. 


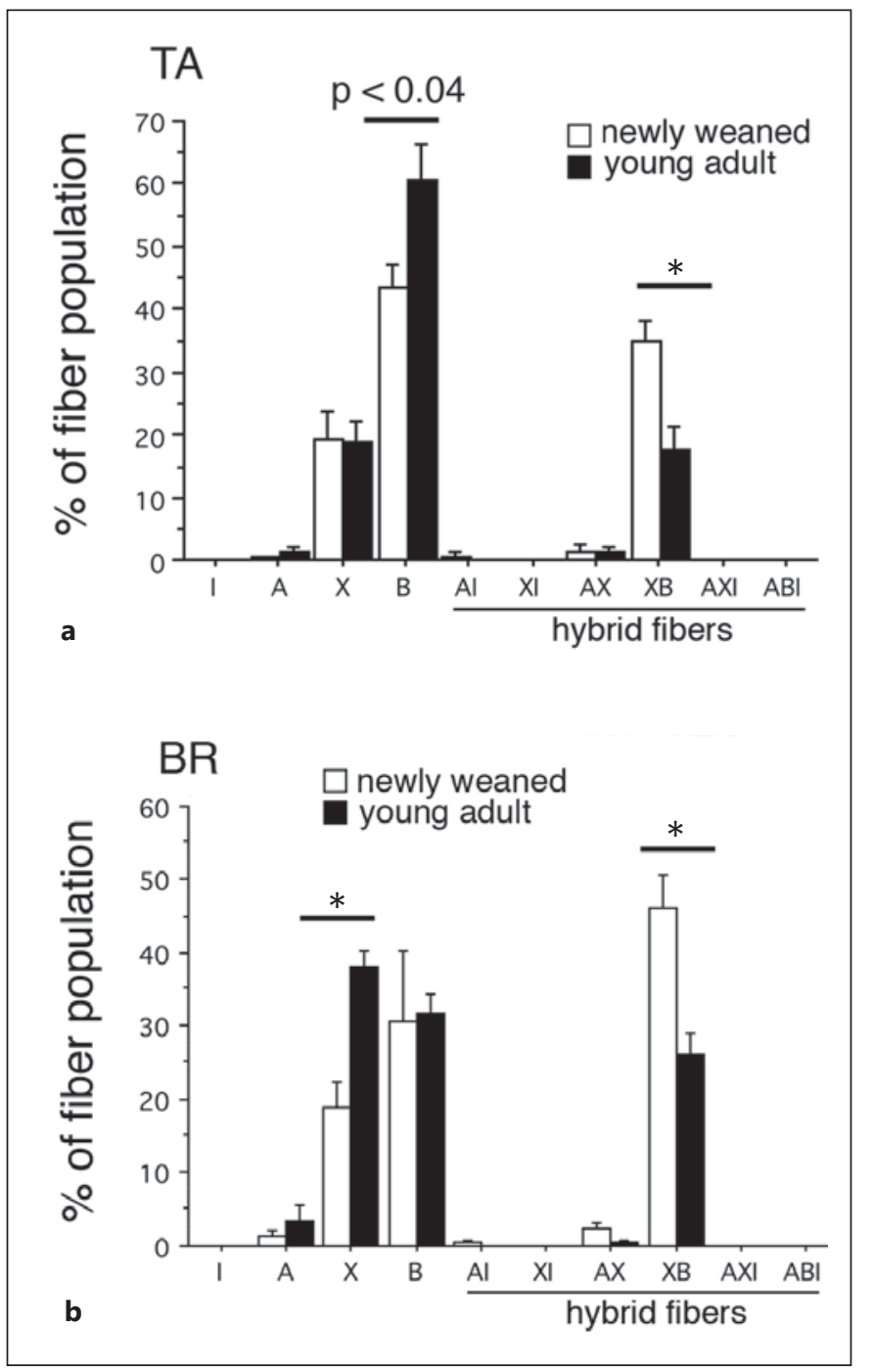

Fig. 4. Fiber type proportions in newly weaned versus young adult mice. a In the TA muscles, the IIX/IIB hybrids are significantly higher in the newly weaned mice than in young adult mice $(\mathrm{p}<$ 0.008 ). The difference in IIB fibers between newly weaned and young adult mice was not statistically significant $(p<0.04)$. b In the BR muscles, IIX/IIB hybrids were significantly fewer in the young adult compared with newly weaned mice $(\mathrm{p}<0.010)$. This lower number of hybrids was coincident with a significantly higher proportion of pure IIX fibers in the older mice $(\mathrm{p}<0.005)$.

Hybrid Fiber Proportion as a Function of Mouse Age

The relative proportions of hybrid fibers were determined from approximately 100 fibers from each muscle sampled (range 71-170 fibers; mean $112 \pm 26$ fibers). The total numbers of all hybrids were combined into a polymorphism index (total proportion of all hybrid fibers [Caiozzo et al., 2003]) which was used for subsequent sta- tistical analyses. In the TA and BR muscles, the polymorphism index was highest in newly weaned mice and then declined in a curvilinear pattern that leveled off with time. As an alternative to mouse age as an independent variable, we used animal mass because this variable is a direct indicator of animal growth (fig. 3). For both the TA and BR muscles, the decline in hybrid fibers was fit with a power regression. For the TA muscles, the slope of the regression was negative and significant using both age $(\mathrm{p}<0.0008)$ and mass $(\mathrm{p}<0.0033)$ as the independent variable. For the BR, the slope was not significant for mouse age $(\mathrm{p}<0.078)$, but was negative and significant for mouse mass $(p<0.028)$. For the SOL there was no significant change in hybrid proportion either as a function of mouse age or mass (fig. 3).

\section{Fate of Hybrid Fibers in TA and BR Muscles}

Given the significant decline in hybrid fibers with maturation in both the TA and BR muscles, a relevant question is: what happened to these fibers? Since the large majority of hybrids in these muscles were IIX/IIB, we focused on the differences in the proportions of these fibers in newly weaned versus mature mice (fig. 4). In the TA muscles, the proportion of IIX/IIB hybrids was significantly lower in mature mice $(34.9 \%$ in newly weaned mice vs. $17.7 \%$ in mature mice; $\mathrm{p}<0.008)$. Coincident with this difference, the IIB fibers were $60.7 \%$ in mature mice versus $43.3 \%$ in newly weaned mice, but this difference was not statistically significant (fig. 4a; $\mathrm{p}<0.04$; Bonferroni-adjusted critical $\mathrm{p}=0.025)$. However, a linear regression analysis revealed that the proportions of IIB and IIX/IIB hybrid fibers were inversely correlated with one another in the TA $\left(\mathrm{r}^{2}=\right.$ $0.522 ; \mathrm{p}<0.028$ ). No correlation was observed between IIX and IIX/IIB hybrids $\left(\mathrm{r}^{2}=0.02 ; \mathrm{p}>0.68\right)$. In the BR muscles, the proportion of IIX/IIB hybrids was also significantly lower in mature mice $(46.3 \%$ in newly weaned mice vs. $26 \%$ in mature mice; $\mathrm{p}<0.01$ ). In the BR muscles the proportion of IIX fibers was significantly greater in mature mice (fig. $4 \mathrm{~b} ; 38 \%$ in mature mice vs. $18.9 \%$ in newly weaned mice; $\mathrm{p}<0.005$ ), while the proportion of IIB fibers was stable. The total pool of IIX, IIB and IIX/IIB fibers represented 97.4 and $95.9 \%$ of the fibers in the TA and BR, respectively. These proportions did not change with maturation ( $t$ tests comparing newly weaned with young adult mice: $p>0.99$ and $p>0.98$ for the TA and BR, respectively). These trends suggest that the IIX/IIB hybrids lost during maturation were predominantly converted into IIB fibers in the TA muscles, but were primarily transformed into IIX fibers in the BR (fig. 5). In both muscles, a significant proportion of stable hybrids ( 20-25\%) were still present in the oldest mice examined. 


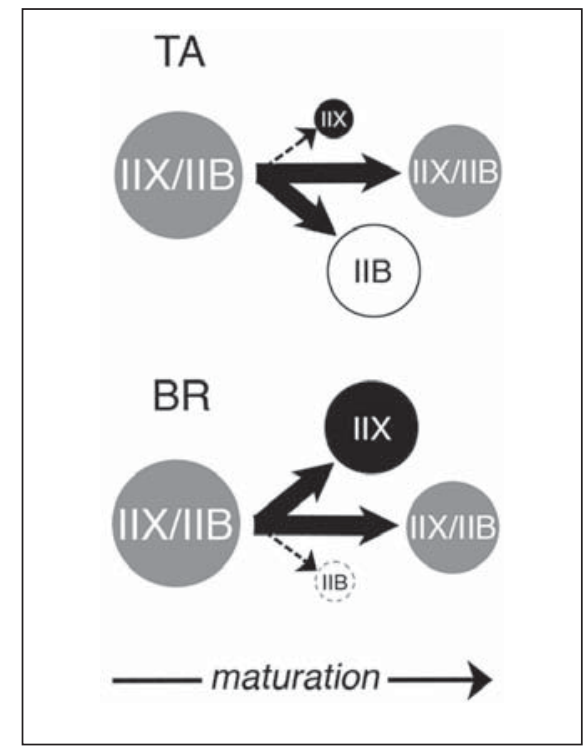

Fig. 5. Proposed role for IIX/IIB hybrids in fiber type transitions. The pool of IIX/IIB hybrids declines with maturation in both the TA and BR. About half of the hybrid fibers persist as phenotypically stable hybrids, while about half transform into either IIB (TA) or IIX (BR) fibers. Minor numbers of hybrid fibers also likely transform into IIX in the TA, and into IIB in the BR.

\section{Proportions of IIX and IIB Isoforms in IIX/IIB}

Hybrids

In a previous study, we determined that IIX/IIB hybrids form a continuum of fiber types in the mouse TA and BR muscles [Zhang et al., 2010]. In the current study, we were interested in whether these proportions changed during muscle maturation, as hybrid fibers were being converted into pure IIX or IIB fibers. Overall, we again observed a continuum of fiber types, from predominantly IIX-containing hybrids to predominantly IIB-containing hybrids. On average, there was an equal proportion of IIX and IIB isoforms and these were normally distributed (fig. 6). In the BR muscles, the average proportion of the IIX isoform contained within these hybrids significantly increased as a function of mouse age ( $\mathrm{p}<0.02)$, possibly indicating a shift toward the IIX fiber type (data not shown). However, the proportion of the IIX isoform was still close to $50 \%$ (range 44-60\%). No significant shift in MHC isoform proportions was observed within IIX/IIB hybrids from the TA.

\section{MHC Isoform Distribution along the Length of Single}

Fibers

We divided single fibers from the TA and BR muscles of newly weaned mice into three equal segments (end,
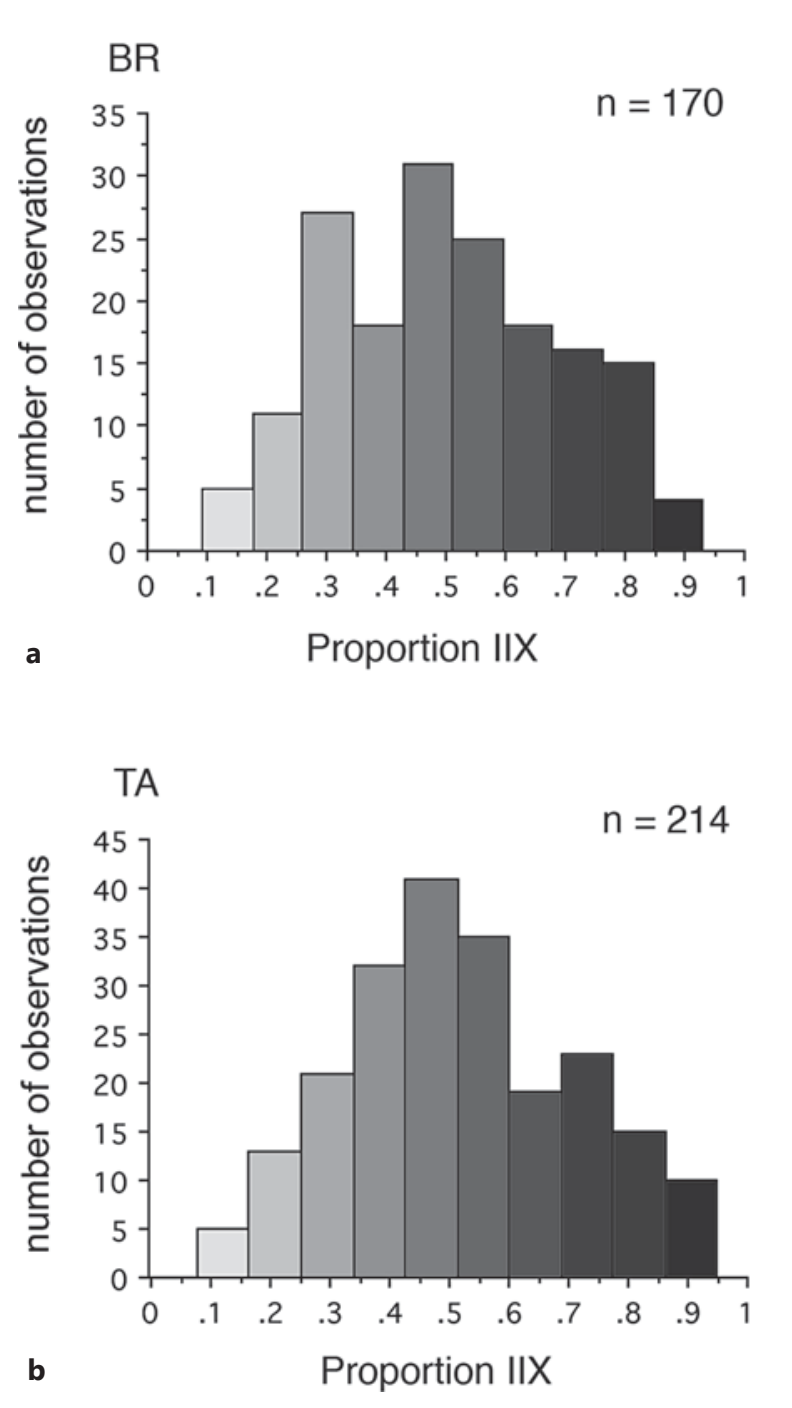

Fig. 6. Relative proportion of the IIX isoform within single IIX/IIB hybrid fibers of the BR (a) and TA (b) muscles. Darker columns indicate a greater proportion of IIX MHC, while the lighter columns correspond to greater amounts of IIB. Overall, the proportions of these isoforms are normally distributed, with the largest group of hybrids containing approximately equal amounts of each isoform.

middle, and end'). MHC isoform content for each segment was then determined using the described SDSPAGE procedure. We observed four patterns of MHC content in these fibers: (1) pure IIX fibers (fig. 7a), (2) pure IIB fibers (fig. 7b), (3) symmetrical IIX/IIB hybrids with similar proportions in each segment (fig. 7c) and (4) asymmetrical IIX/IIB hybrids where the proportions of the IIX and IIB isoforms differed along the length of the 
fiber (fig. 7e-g). Of the 48 fibers studied, $23 \%$ were pure IIX, $42 \%$ were pure IIB, $10 \%$ were symmetrical IIX/IIB hybrids and $25 \%$ were asymmetrical IIX/IIB hybrids. We quantified the relative proportions of IIX and IIB isoforms within these segments of single asymmetrical hybrid fibers and found that a greater proportion of the IIX isoform was concentrated at one end (fig. 8). Generally, there was a gradation from greater IIX content toward one end and mostly IIB toward the other (fig. 8).

\section{Discussion}

\section{Fiber Type Transitions with Maturation}

In the current study, analyses of single fiber $\mathrm{MHC}$ isoform content revealed that the muscles of young mice experience significant changes in fiber type well past the time of weaning. In both the BR and TA muscles, the proportion of hybrid fibers was close to $50 \%$ shortly after weaning, but declined to approximately half that value over the next several weeks and months (fig. 3). In the TA muscle, the decline in IIX/IIB hybrids occurred in synchrony with increased IIB content, while in the BR muscle the reduction of IIX/IIB fibers was accompanied by an increase in IIX fibers. Our interpretation of these data is that about half of the IIX/IIB hybrids persisted as hybrids in both muscles, but the other half were converted into IIX fibers in the BR, while similar proportions were converted into IIB fibers in the TA (fig. 5). These patterns show that the fate of hybrid fibers in fiber type transitions can be complex, as the same hybrid fiber types (IIX/IIB) in different muscles may be converted into distinct 'pure' fiber types (either IIX or IIB) during the same stages of maturation. However, not all of the hybrids in the three muscles studied were transitional, since the proportion of hybrids in the SOL was a steady $20 \%$ of fibers in the muscles of mice across all ages, and $20-25 \%$ of all fibers were hybrids in the BR and TA at 6 months of age (fig. 3,4 ). These persistent populations of hybrid fibers in mouse muscles are consistent with the common occurrence of hybrid fibers in normal muscles from a variety of species [Unguez et al., 2000; Lutz et al., 2001; Stephenson, 2001; Caiozzo et al., 2003; Medler et al., 2004; Strbenc et al., 2006].

The precise roles of hybrid fibers in skeletal muscle plasticity are incompletely understood [Stephenson, 2001; Caiozzo, 2002; Caiozzo et al., 2003], but our results show that they do play a role as intermediate fibers during fiber type transitions (fig. 5). Any fibers experiencing changes in MHC expression must become hybrids for a

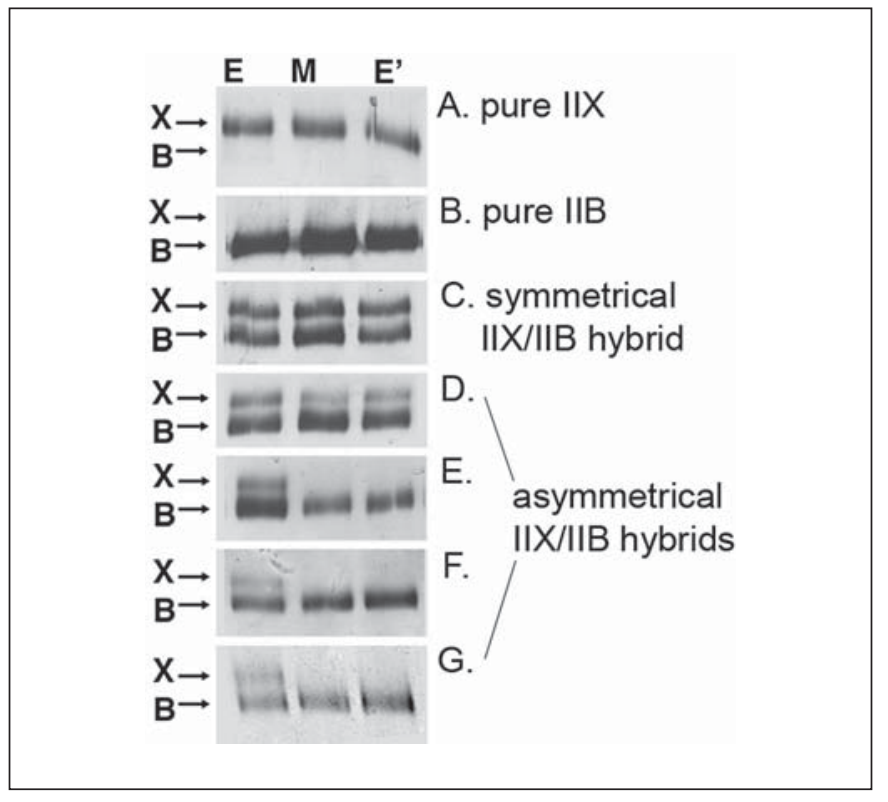

Fig. 7. Proportions of IIX and IIB isoforms in fiber segments along the length of single fibers. Fibers were divided into three segments [two ends (E and E') and a middle (M)]. In pure IIX (A) or IIB fibers (B), MHC expression was uniform among the different fiber regions. Similarly, some of the IIX/IIB hybrids exhibited symmetrical mixtures of both isoforms along the fiber length (C). In $65 \%$ of the IIX/IIB hybrids, we observed asymmetrical distribution of the $\mathrm{MHC}$ isoforms present (D-G).

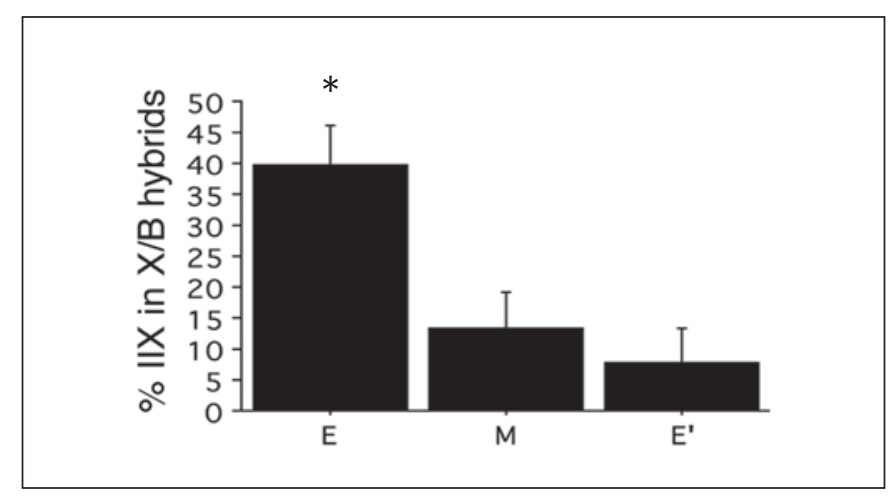

Fig. 8. Significant differences exist in MHC proportions among segments of single fibers. Relative proportions of IIX and IIB isoforms from segments of single fibers (see fig. 7) were quantified using densitometry. Among asymmetric IIX/IIB hybrids, a significant concentration of type IIX MHC was located at one end of single fibers $(\mathrm{n}=12$ fibers; $\mathrm{p}<0.0014)$. 
period of time, as the obsolete isoforms are removed and the new ones are incorporated into the myofibrils. This role is particularly important during early muscle development and subsequent postnatal maturation, when dramatic shifts in fiber type are taking place [Di Maso et al., 2000; Unguez et al., 2000; Strbenc et al., 2006]. During fetal development, skeletal muscle fibers express the embryonic MHC isoform exclusively, or in combination with other isoforms [Di Maso et al., 2000; Agbulut et al., 2003; Lieber, 2010]. Next, embryonic MHC is exchanged for neonatal MHC, which is then expressed in conjunction with adult isoforms [Di Maso et al., 2000; Agbulut et al., 2003; Lieber, 2010]. By the time of birth, most muscle fibers express some levels of embryonic and neonatal isoforms in various combinations with one another or with adult isoforms [Di Maso et al., 2000; Unguez et al., 2000; Strbenc et al., 2006; Wank et al., 2006]. Over the subsequent weeks following birth, these developmental isoforms are quickly lost and adult MHC isoform expression increases dramatically. In mouse muscles, the developmental MHC isoforms are almost completely absent from the muscles by the time of weaning (day 21) [Agbulut et al., 2003]. However, our results demonstrate that the relative proportions of these adult MHC isoforms continue to be in flux for the next several weeks or months in mouse muscles. This pattern of a continued, gradual transition into mature fiber types is consistent with similar changes in the muscles of other species [Wigston and English, 1992; Unguez et al., 2000; Strbenc et al., 2006; Medler et al., 2007].

The first several weeks after birth represent a period of major growth and differentiation of skeletal muscles [Agbulut et al., 2003; Wank et al., 2006; Gokhin et al., 2008; White et al., 2010]. These changes are multifaceted and dramatic, affecting not only fiber type, but also changes in muscle mass, metabolic enzymes, specific tension and excitation-contraction coupling [Wank et al., 2006; Gokhin et al., 2008; Goodman et al., 2008; White et al., 2010]. Since fiber number is essentially fixed at birth, increases in muscle mass and transitions in muscle fiber type must be accomplished by remodeling existing fibers. Increases in mass occur as greater densities of myofibrils are packed into fibers, and as fibers grow in length [Gokhin et al., 2008; Goodman et al., 2008; White et al., 2010]. Not only are fibers growing in size, but the specific tension generated by each fiber also increases because of more dense packing of myofibrils within each fiber [Gokhin et al., 2008; Goodman et al., 2008]. These changes in fiber mass and myofibrillar packing go hand in hand with fiber type transitions. Shifts in fiber type require in- creased expression and incorporation of new isoforms, as well as the removal of obsolete MHC isoforms. In mouse muscles, adult MHC isoforms are expressed by the time of weaning, but significant changes in the expression of these isoforms within single fibers continues as they incorporate new myofibrils into their growing muscles. When the MHC in these new myofibrils is different from the $\mathrm{MHC}$ in the existing myofibrils, the result is a change in fiber type.

\section{Segmental Differences in Fiber Type}

In a previous study, we compared the MHC content in adjacent fiber segments from the TA and BR muscles and found that asymmetries were common [Zhang et al., 2010]. In the current study, we found that $2 / 3$ of the IIX/ IIB hybrids from newly weaned mice exhibited asymmetries in MHC isoform content along the length of single fibers (fig. 7, 8). Although the conventional view is that individual muscle fibers are composed of a single fiber type and, therefore, contract as a coordinated unit, the existence of fiber type asymmetries draws this basic assumption into question. Edman et al. [1985] discovered significant differences in shortening velocity among segments within single frog muscle fibers and proposed that these differences resulted from differential expression of $\mathrm{MHC}$ isoforms in different fiber regions. These regional differences in MHC content within single frog fibers and their contribution to differences in intra-fiber contractile properties have since been confirmed [Edman et al., 1988; Lutz et al., 2001]. More recently, independent patterns of muscle strain within single fascicles have been identified in toad muscles in vivo [Ahn et al., 2003]. Although the functional significance of regional differences in muscle contraction is poorly understood, we can propose a mechanism for their occurrence.

Muscle fibers are enormous cells, formed by the fusion of individual myoblasts, and are often several millimeters in length [Lieber, 2010]. The presence of hundreds of individual nuclei, along with limitations in diffusional distance, means that the different myonuclear domains can contain distinct protein assemblages from one another [Pavlath et al., 1989; Newlands et al., 1998; Allen et al., 1999]. Indeed, a number of studies have demonstrated that myosin expression does vary along the length within certain single muscle fibers [Staron and Pette, 1987; Edman et al., 1988; Termin et al., 1989; Sakuma et al., 1995; Peuker and Pette, 1997; Lutz et al., 2001; Zhang et al., 2010]. While it is currently unknown how prevalent such asymmetries are in normal muscles, it is plausible that longitudinal differences in MHC content may reflect dy- 
namic changes in fiber type. For example, segmental differences in MHC expression could be related to dramatic increases in muscle fiber length that take place during the rapid muscle growth phase following weaning [White et al., 2010]. In maturing rat SOL muscles, regional asymmetries in fiber type are present in single fibers between 3 and 4 weeks of age, but these fibers then become more homogeneous over time [Sakuma et al., 1995]. In developing bird muscles, neonatal MHC is expressed at the growing ends of the fibers, but adult MHCs are found at the muscle mid-regions [Rosser et al., 1995]. We found that in many cases, one end of the fiber differed in MHC content as compared with the rest of the fiber (fig. 7, 8). Since the muscles of newly weaned mice grow rapidly at their ends [White et al., 2010], one explanation for these differences is that the MHC isoform incorporated into the myofibrils at the regions of growth may differ from the MHC in the existing fiber regions. Further work is needed to better define the role of muscle growth in producing fiber asymmetries.

In conclusion, we found that IIX/IIB hybrid fibers represent approximately $40-50 \%$ of fibers in the TA and
BR of newly weaned mice, but these hybrids are converted into pure IIX and IIB fiber types over the subsequent weeks and months. Therefore, many of the hybrids in these muscles are transitional fiber types in maturing mouse muscles. Many of the hybrids within these muscles exhibit pronounced asymmetries in MHC content along their lengths, a pattern that raises a number of important questions for muscle fiber type regulation. We propose that regional asymmetries may arise during the rapid growth in fiber length, when the newly incorporated MHC isoform may differ from the existing ones. Further research is needed to address the role of these $\mathrm{MHC}$ asymmetries during muscle growth and development.

\section{Acknowledgements}

This work was supported by the National Institute of Arthritis and Musculoskeletal and Skin Diseases grant RAR-053666A to S. Medler. We sincerely appreciate the insightful comments from two anonymous reviewers of the manuscript.

\section{References}

Acevedo, L.M., J.L. Rivero (2006) New insights into skeletal muscle fibre types in the dog with particular focus towards hybrid myosin phenotypes. Cell Tissue Res 323: 283-303.

Agbulut, O., P. Noirez, F. Beaumont, G. ButlerBrowne (2003) Myosin heavy chain isoforms in postnatal muscle development of mice. Biol Cell 95: 399-406.

Ahn, A.N., R.J. Monti, A.A. Biewener (2003) In vivo and in vitro heterogeneity of segment length changes in the semimembranosus muscle of the toad. J Physiol 549: 877-888.

Allen, D.L., R.R. Roy, V.R. Edgerton (1999) Myonuclear domains in muscle adaptation and disease. Muscle Nerve 22: 1350-1360.

Andersen, J.L. (2003) Muscle fibre type adaptation in the elderly human muscle. Scand J Med Sci Sports 13: 40-47.

Andersen, J.L., G. Terzis, A. Kryger (1999) Increase in the degree of coexpression of myosin heavy chain isoforms in skeletal muscle fibers of the very old. Muscle Nerve 22: 449-454.

-Blough, E., E. Rennie, F. Zhang, P. Reiser (1996) Enhanced electrophoretic separation and resolution of myosin heavy chains in mammalian and avian skeletal muscles. Anal Biochem 233: 31-35.

-Caiozzo, V.J. (2002) Plasticity of skeletal muscle phenotype: mechanical consequences. Muscle Nerve 26: 740-768.

Caiozzo, V.J., M.J. Baker, K. Huang, H. Chou, Y. Wu, K. Baldwin (2003) Single-fiber myosin heavy chain polymorphism: how many patterns and what proportions? Am J Physiol Regul Integr Comp Physiol 285: R570-R580.

Di Maso, N.A., V.J. Caiozzo, K.M. Baldwin (2000) Single-fiber myosin heavy chain polymorphism during postnatal development: modulation by hypothyroidism. Am J Physiol Regul Integr Comp Physiol 278: R1099-R1106.

Edman, K.A.P., C. Reggiani, S. Schiaffino, G. te Kronnie (1988) Maximum velocity of shortening related to myosin isoform composition in frog skeletal-muscle fibers. J Physiol 395: 679-694.

Edman, K.A.P., C. Reggiani, G. te Kronnie (1985) Differences in maximum velocity of shortening along single muscle-fibers of the frog. J Physiol 365: 147-163.

Glaser, B., G. You, M. Zhang, S. Medler (2010) Relative proportions of hybrid fibers are unaffected by 6 weeks of running exercise in mouse skeletal muscles. Exp Physiol 95: 211221.

Gokhin, D.S., S.R. Ward, S.N. Bremner, R.L. Lieber (2008) Quantitative analysis of neonatal skeletal muscle functional improvement in the mouse. J Exp Biol 211: 837-843.

Goodman, C.A., R. Blazev, J. Kemp, G.M.M. Stephenson (2008) E-C coupling and contractile characteristics of mechanically skinned single fibres from young rats during rapid growth and maturation. Pflügers Arch 456: 12171228 .
Lieber, R.L. (2010) Skeletal Muscle Structure, Function, and Plasticity: the Physiological Basis of Rehabilitation. Baltimore, Lippincott Williams and Wilkins.

Lutz, G.J., S.N. Bremner, M.J. Bade, R.L. Lieber (2001) Identification of myosin light chains in Rana pipiens skeletal muscle and their expression patterns along single fibres. J Exp Biol 204: 4237-4248.

Medler, S., T. Lilley, D.L. Mykles (2004) Fiber polymorphism in skeletal muscles of the American lobster, Homarus americanus: continuum between slow-twitch (S-1) and slowtonic (S-2) fibers. J Exp Biol 207: 2755-2767.

Medler, S., T.R. Lilley, J.H. Riehl, E.P. Mulder, E.S Chang, D.L. Mykles (2007) Myofibrillar gene expression in differentiating lobster claw muscles. J Exp Zool A Ecol Genet Physiol 307A: 281-295

Medler, S., D.L. Mykles (2003) Analysis of myofibrillar proteins and transcripts in adult skeletal muscles of the American lobster Homarus americanus: variable expression of myosins, actin and troponins in fast, slow-twitch and slow-tonic fibres. J Exp Biol 206: 3557-3567.

Newlands, S., L.K. Levitt, C.S. Robinson, A.B.C. Karpf, V.R.M. Hodgson, R.P. Wade, E.C. Hardeman (1998) Transcription occurs in pulses in muscle fibers. Genes Dev 12: 2748-2758.

Pavlath, G.K., K. Rich, S.G. Webster, H.M. Blau (1989) Localization of muscle gene-products in nuclear domains. Nature 337: 570-573. 
Pette, D., R.S. Staron (2001) Transitions of muscle fiber phenotypic profiles. Histochem Cell Biol 115: 359-372.

Peuker, H., D. Pette (1997) Quantitative analyses of myosin heavy-chain mRNA and protein isoforms in single fibers reveal a pronounced fiber heterogeneity in normal rabbit muscles. Eur J Biochem 247: 30-36.

Rosser, B.W.C., D.M. Waldbillig, S.D. Lovo, J.D. Armstrong, E. Bandman (1995) Myosin heavy-chain expression within the tapered ends of skeletal-muscle fibers. Anat Rec 242: 462-470.

Sakuma, K., A. Yamaguchi, H. Ohmori, S. Katsuta (1995) Nonuniform changes in fiber types in the soleus muscle of the developing rat. Eur J Appl Physiol Occup Physiol 70: 132137.

-Schiaffino, S., C. Reggiani (1996) Molecular diversity of myofibrillar proteins: gene regulation and functional significance. Physiol Rev 76: 371-423.

Schiaffino, S., C. Reggiani (2011) Fiber types in mammalian skeletal muscles. Physiol Rev 91: 1447-1531.

-Staron, R.S., D. Pette (1987) Nonuniform myosin expression along single fibers of chronically stimulated and contralateral rabbit tibialis anterior muscles. Pflügers Arch 409: 67-73.
-Staron, R.S., D. Pette (1993) The continuum of pure and hybrid myosin heavy chain-based fiber types in rat skeletal muscle. Histochemistry 100: 149-153.

Stephenson, G.M.M. (2001) Hybrid skeletal muscle fibres: a rare or common phenomenon? Clin Exp Pharmacol Physiol 28: 692-702.

-Strbenc, M., V. Smerdu, A. Pogacnik, G. Fazarinc (2006) Myosin heavy chain isoform transitions in canine skeletal muscles during postnatal growth. J Anat 209: 149-163.

Talmadge, R.J., R.R. Roy (1993) Electrophoretic separation of rat skeletal muscle myosin heavy chain isoforms. J Appl Physiol 75: 2337-2340.

Talmadge, R.J., R.R. Roy, V.R. Edgerton (1995) Prominence of myosin heavy-chain hybrid fibers in soleus muscle of spinal cord-transected rats. J Appl Physiol 78: 1256-1265.

Talmadge, R.J., R.R. Roy, V.R. Edgerton (1999) Persistence of hybrid fibers in rat soleus after spinal cord transection. Anat Rec 255: 188201.

Termin, A., R.S. Staron, D. Pette (1989) Changes in myosin heavy chain isoforms during chronic low-frequency stimulation of rat fast hindlimb muscles: a single-fiber study. Eur J Biochem 186: 749-754.

Unguez, G.A., R.J. Talmadge, R.R. Roy, D. Dalponte, V.R. Edgerton (2000) Distinct myosin heavy chain isoform transitions in developing slow and fast cat hindlimb muscles. Cells Tissues Organs 167: 138-152.
Wank, V., M.S. Fischer, B. Walter, R. Bauer (2006) Muscle growth and fiber type composition in hind limb muscles during postnatal development in pigs. Cells Tissues Organs 182: 171-181.

White, R.B., A.S. Bierinx, V.F. Gnocchi, P.S. Zammit (2010) Dynamics of muscle fibre growth during postnatal mouse development. BMC Dev Biol 10: 21.

Wigston, D.J., A.W. English (1992) Fiber-type proportions in mammalian soleus muscle during postnatal development. J Neurobiol 23: 61-70.

Williamson, D.L., M.P. Godard, D.A. Porter, D.L. Costill, S.W. Trappe (2000) Progressive resistance training reduces myosin heavy chain coexpression in single muscle fibers from older men. J Appl Physiol 88: 627-633.

Wray, W., T. Boulikas, V.P. Wray, R. Hancock (1981) Silver staining of proteins in polyacrylamide gels. Anal Biochem 118: 197-203.

Zhang, M.Y., W.J. Zhang, S. Medler (2010) The continuum of hybrid IIX/IIB fibers in normal mouse muscles: $\mathrm{MHC}$ isoform proportions and spatial distribution within single fibers. Am J Physiol Regul Integr Comp Physiol 299: 1582-1591. 